\title{
Comentario al artículo “Estudio económico del tratamiento de las quemaduras térmicas mediante deshridamiento enzimático; papel determinante de la estancia hospitalaria"
}

\author{
Eugenia LÓPEZ-SUSO \\ Cirujano Plástico, Coordinadora de la Unidad de Quemados, Complejo Hospital Universitario A Coruña, A Coruña, España.
}

El trabajo de Martínez y col. muestra que, desde la perspectiva del Hospital La Paz (Madrid-España), el coste sanitario durante la hospitalización de los pacientes que han sobrevivido al daño térmico se incrementa significativamente con la extensión y con la profundidad de las quemaduras. Los pacientes que sobreviven a quemaduras $>15 \%$ tienen un gasto sanitario medio durante la hospitalización de unos $60.000 €$. En los subgrupos de pacientes que analiza, el porcentaje de coste atribuido al tratamiento con Nexobrid ${ }^{\circledR}$ se reduce a medida que la necesidad de otros recursos sanitarios es mayor. Sus resultados merecen alguna reflexión:

Primero: los pacientes quemados que precisan ingreso en una Unidad de Referencia requieren recursos y el apoyo del programa si el hospital desea ofrecer atención o, en el caso de que se contemple la expansión de servicios, debe ser económicamente viable o cumplir con el objetivo de la institución. En España, las Unidades de Referencia de Quemados Críticos se han definido como el dispositivo asistencial de alta especialización y complejidad, integrado en un centro hospitalario, dotado con los recursos humanos y materiales necesarios para la atención sanitaria basada en la mejor evidencia disponible de este tipo de pacientes. La regionalización de la atención médica se ha convertido en un tema importante en muchos países, y es necesario entender la economía que hay detrás de este movimiento.

Segundo: para tratar de ubicar los aspectos financieros relacionados con la atención a pacientes quemados críticos en una perspectiva administrativa, podemos compararlos con el coste de la atención hospitalaria brindada a los pacientes con parada cardíaca extrahospitalaria, o con infarto de miocardio con elevación del ST, patologías en las que comúnmente se considera que hay un uso apropiado y valioso de los recursos. La literatura ${ }^{(1)}$ muestra un costo similar, por lo que los hospitales que atienden a pacientes quemados críticos como Unidades de Referencia no soportan una carga financiera "indebida".

Tercero: la evaluación económica de una intervención sanitaria conjuga 2 elementos en su análisis. Por una parte el coste, importante sin duda, pero por otra parte también la medida de los resultados de las intervenciones, tanto o más crucial si cabe. El problema radica en la dificultad, o incluso en la imposibilidad, de asignar un valor económico a los beneficios en el área de la salud que refleje de forma válida los múltiples matices que un resultado clínico puede albergar. Los autores y editores de revistas biomédicas deben hacer el esfuerzo de agregar ambas corrientes de información y difundir una cultura de la evaluación de las intervenciones sanitarias basada en la plena integración de costes y resultados. ${ }^{(2)}$

Bibliografía

1. Swor R, Lucia V, McQueen K, Compton S. Hospital costs and revenue are similar for resuscitated out-of-hospital cardiac arrest and ST-segment acute myocardial infarction patients. Acad Emerg Med. 2010;17(6):612-616.

2. Prieto L, Sacristán JA, Pinto JL, Badia X, Antoñanzas F, del Llano J; Grupo ECOMED. Analysis of costs and results of the economic assessment of health interventions. Med Clin (Barc). 2004,27;122(11):423-429. 